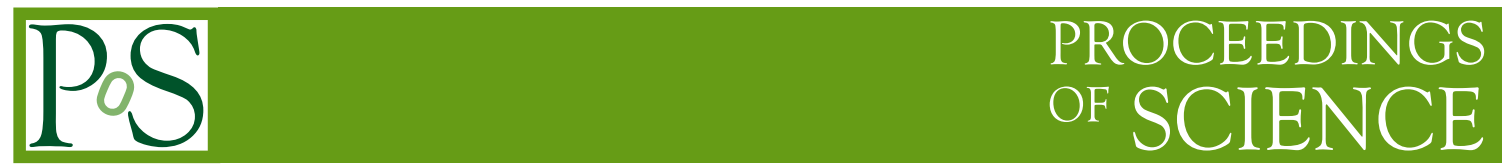

\title{
The quark-gluon plasma in an external magnetic field
}

\section{Ludmila Levkova and Carleton DeTar*}

Department of Physics and Astronomy, University of Utah, Salt Lake City, UT 84112-0830, USA

E-mail: ludmila@physics.utah.edu, detar@physics.utah.edu

\begin{abstract}
We present results of an exploratory study of the effect of a strong magnetic field on the equation of state of high temperature strongly interacting matter. Working with conventional gauge field ensembles at zero magnetic field, we compute terms up to second order in a Taylor series expansion of the pressure as a function of the magnetic field.
\end{abstract}

31st International Symposium on Lattice Field Theory - LATTICE 2013

July 29 - August 3, 2013

Mainz, Germany

\footnotetext{
* Speaker.
} 


\section{Introduction}

Conditions that create a quark-gluon plasma could also result in strong (electromagnetic) fields of order $O\left(10^{14-15}\right) \mathrm{T}$. (In GeV units $0.2 \mathrm{GeV}^{2} / e \approx 3 \times 10^{15} \mathrm{~T}$.) Examples are a noncentral heavy ion collision or the primordial quark gluon plasma in the early universe. Such fields could alter the crossover temperature, the equation of state, and other properties of the plasma.

Since our most reliable theoretical understanding about the properties of the quark-gluon plasma in thermal equilabrium and low quark chemical potential comes from numerical lattice QCD simulations, there has been a recent surge of interest in studying the thermal effects of such fields. Work so far has focused on the chiral properties, e.g. $\langle\bar{\psi} \psi\rangle$ and the effect on $T_{c}$ $[1,2,3,4,5,6,7]$. These studies have required generating dedicated gauge field ensembles with an explicit external $B$ field. Our exploratory study aims to calculate the equation of state using lattice QCD [8].

\section{Taylor expansions}

In our study we employ a Taylor expansion of the thermodynamic potential as a function of the strength of the external $B$ field to approximate the change in pressure due to the field. This approach eliminates the need to generate dedicated gauge field ensembles with different $B$ :

$$
\begin{aligned}
\frac{p(T)}{T^{4}} & =\frac{\ln Z(B)}{T^{3} V}=\sum_{n=0}^{\infty} C_{n}(T)\left(|e| B / T^{2}\right)^{n} \\
C_{n}(T) & =\left.\frac{L_{t}^{3}}{L_{s}^{3}} \frac{1}{n !} \frac{\partial^{n} \ln Z(B)}{\partial\left(|e| B / T^{2}\right)^{n}}\right|_{B=0} .
\end{aligned}
$$

Only even terms are nonzero because of CP symmetry. The first nontrivial term (second-order) is ultraviolet divergent, so a zero temperature subtraction is needed. This step also renormalizes the electric charge [9]. Not much is known about the convergence of the expansion, but D'Elia et al. calculated $\langle\bar{\psi} \psi\rangle$ and found that for $e B \lesssim 0.7 \mathrm{GeV}^{2}$, the $O\left(B^{4}\right)$ correction is small $[1,2]$ However, this calculation was done on a rather coarse lattice with fairly heavy quarks.

Lattice simulations are typically done on a torus. The convetional approach introduces a constant uniform field throughout the lattice volume. To achieve uniformity and keep periodic boundary conditions on the torus, it is necessary to quantize the field. For example with constant $\vec{B}=B \hat{z}$, torus quantization with (quark charge $|q|=|e| / 3)$ requires $|q| B=2 \pi b /\left(L_{x} L_{y} a^{2}\right)$ with integer $b \in\left(0, L_{x} L_{y} / 2\right)$. A typical choice for the continuum vector potential is

$$
A_{y}=B x, \quad A_{\mu}=0 \quad \text { for } \quad \mu=\hat{x}, \hat{z}, \hat{t} .
$$

On the lattice one can choose $U(1)$ links so that the $y$ links are

$$
u_{y}(B, q, X)=e^{i a^{2} q B x}
$$

for four-coordinate $X=(x, y, z, t)$, and the other links are

$$
u_{x, z, t}(B, q, X)= \begin{cases}1 & \text { for } x \in\left[0, L_{x}-2\right] \\ e^{-i a^{2} q B L_{x} y} & \text { for } x=L_{x}-1 .\end{cases}
$$

But quantization is unsuitable for a Taylor expansion. If the quantization condition is not met, the field cannot be made uniform, which has a profound effect on physical observables. 


\section{Half and half}

The remedy we have been exploring introduces a uniform (positive) flux in half the lattice, and the opposite flux in the other half:

$$
B_{z}(x)=\left\{\begin{array}{l}
+B \text { for } x<L_{x} / 2 \\
-B \text { for } x \geq L_{x} / 2
\end{array} .\right.
$$

Because the return flux is introduced explicitly in a uniform way, no quantization is needed. In effect, this set up introduces a constant electic current sheet at the two surfaces where the flux reverses. Since physical phenomena are independent of the direction of the field, we expect that, except for the boundary contribution, the two halves should contribute equally to changes in the pressure, and the modified boundary condition should be harmless for sufficiently large volumes. Of course, it becomes especially important to check for finite volume effects, which are probably $O\left(1 / L_{x}\right)$

We use the HISQ action with $2+1$ flavors. The partition function is

$$
Z(B)=\int d U e^{-S_{g}} e^{\frac{1}{4} \ln \operatorname{det} M^{u}\left(B, q_{u}\right)} e^{\frac{1}{4} \ln \operatorname{det} M^{d}\left(B, q_{d}\right)} e^{\frac{1}{4} \ln \operatorname{det} M^{s}\left(B, q_{s}\right)} .
$$

where $M^{f}$ is the HISQ/asqtad fermion matrix for flavor $f$, namely

$$
M_{X, Y}^{f}\left(B, q_{f}\right)=a m_{f} \delta_{X, Y}+D_{X, Y}^{z, t, x}+D_{X, Y}^{y}\left(B, q_{f}\right)
$$

where the $B$-independent term $D_{X, Y}^{z, t, x}$ is a sum of the Dirac operators in the $\hat{x}, \hat{z}$ and $\hat{t}$ directions at all points. The third term includes the $U(1)$ field $\left(x^{\prime}=x-L_{x} / 4\right.$ for $x \leq L_{x} / 2$, and $x^{\prime}=3 L_{x} / 4-x$ when $x>L_{x} / 2$ ):

$$
\begin{aligned}
D_{X, Y}^{y}\left(B, q_{f}\right)= & \frac{1}{2} \eta_{y}(X)\left[U_{y}^{(F)}(X) \mathrm{e}^{i q_{f} a^{2} B x^{\prime}} \delta_{X+\hat{y}, Y}\right. \\
& \left.+U_{y}^{(L)}(X) \mathrm{e}^{3 i q_{f} a^{2} B x^{\prime}} \delta_{X+3 \hat{y}, Y}-h . c .\right]
\end{aligned}
$$

Terms that depend on the $B$ field are highlighted in blue.

The coefficients of the Taylor expansion are obtained from derivatives of the partition function:

$$
\begin{aligned}
& \partial^{n} \ln \operatorname{det} M_{f} /\left(\partial a^{2} B\right)^{n} \\
& \partial^{n} \operatorname{Tr} M_{f}^{-1} /\left(\partial a^{2} B\right)^{n} \quad \text { (for the interaction measure). }
\end{aligned}
$$

They are, in turn, computed in terms of derivatives of the fermion matrix:

$$
\begin{aligned}
\left.\frac{\partial^{n} M^{f}\left(B, q_{f}\right)}{\partial a^{2} B^{n}}\right|_{B=0} & =\frac{1}{2} \eta_{y}(X)\left[\left(i q_{f} x^{\prime}\right)^{n} U_{y}^{(F)}(X) \delta_{X+\hat{y}, Y}\right. \\
& \left.+\left(3 i q_{f} x^{\prime}\right)^{n} U_{y}^{(L)}(X) \delta_{X+3 \hat{y}, Y}-\text { h.c. }\right] .
\end{aligned}
$$

Traces of these terms are calculated using stochastic estimators. The Taylor coefficients are then assembled "off line." 
Table 1: Results for the subtracted Taylor expansion coefficients. Listed are the temperature in $\mathrm{MeV}$, the gauge coupling $\beta=10 / g^{2}$, the ratio of light to strange sea quark masses, the lattice dimensions at nonzero and "zero" temperature, the number of random sources for the stochastic sample, and the subtracted firstand second-order Taylor expansion coefficients.

\begin{tabular}{|c|c|c|c|c|c|r|r|r|}
\hline$T$ & $\beta$ & $m_{l} / m_{s}$ & $V_{T \neq 0}$ & $V_{T=0}$ & \multicolumn{2}{|c|}{ Rand. sources } & $C_{1}^{r} \times 10^{-4}$ & $C_{2}^{r} \times 10^{-3}$ \\
& & & & & $T \neq 0$ & $T=0$ & & \\
\hline 134 & 6.195 & $0.00440 / 0.0880$ & $32^{3} \times 8$ & $32^{3} \times 32$ & 2400 & 400 & $-1(7)$ & $-0.3(5)$ \\
154 & 6.341 & $0.00370 / 0.0740$ & $32^{3} \times 8$ & $32^{3} \times 32$ & 2400 & 500 & $3(7)$ & $0.4(4)$ \\
167 & 6.423 & $0.00335 / 0.0670$ & $32^{3} \times 8$ & $32^{3} \times 32$ & 1200 & 200 & $-4(7)$ & $2.2(5)$ \\
167 & 6.423 & $0.00335 / 0.0670$ & $48^{3} \times 8$ & $48^{3} \times 48$ & 1200 & 400 & $-9(4)$ & $2.3(5)$ \\
173 & 6.460 & $0.00320 / 0.0640$ & $32^{3} \times 8$ & $32^{3} \times 64$ & 1200 & 200 & $-6(8)$ & $3.8(6)$ \\
227 & 6.740 & $0.00238 / 0.0476$ & $32^{3} \times 8$ & $48^{3} \times 48$ & 1200 & 200 & $-4(3)$ & $10.4(8)$ \\
373 & 7.280 & $0.00142 / 0.0284$ & $32^{3} \times 8$ & $48^{3} \times 64$ & 1200 & 40 & $0(7)$ & $19.3(1.3)$ \\
\hline
\end{tabular}

\section{Some technical details}

We discuss two technical issues. The first has to do with toroidal restrictions on gauge invariance. There are many ways to define a vector potential for the same $B$ field. On a torus these choices define particular values for Polyakov loops. For example, with $A_{y}=B x$, when we integrate along a loop at fixed $x$ that closes at the $y$ boundary, we have $\int A_{y} d y=B L_{y} x$. Other choices of vector potential, such as $A_{y}=B\left(x-x_{0}\right)$ for nonzero $x_{0}$ give different values for the Polyakov loop, but the same $B$ field. Since the Polyakov loop is gauge invariant, these two vector potential fields are not gauge equivalent, and can result in slightly different results for physical quantities. The difference amounts to a change in boundary condition, and it is expected to become insignificant for large volumes. In this example the difference is expected to decrease as $\exp \left(-M_{\pi} L_{y}\right)$.

The second technical issue has to do with charge renomalization. The vacuum pressure depends on $B$. The zero temperature (vacuum) $O\left(B^{2}\right)$ term (divergent when $a \rightarrow 0$ ) renormalizes the electric charge, so we need at least to subtract it from the divergent nonzero temperature $O\left(B^{2}\right)$ term [9]. We go further and just calculate the thermal contribution to the pressure change, which removes the vacuum pressure entirely.

$$
\begin{aligned}
\Delta p(B, T) & =p(B, T)-p(0, T)-p(B, 0)+p(0,0) \\
& =C_{2}^{r}(T)(e B)^{2}+C_{4}^{r}(T)(e B)^{4} / T^{4}+\ldots,
\end{aligned}
$$

where $C_{n}^{r}(T)=C_{n}(T)-C_{n}(0)$.

\section{Results}

We present results of an exploratory calculation using a $2+1$ flavor HISQ plus tree-level Symanzik gauge action. Most of the gauge configurations in the study were provided by the HotQCD collaboration. The lattice scale for these configurations is known [10]. We follow the line of constant physics at fixed $N_{t}=8$ with sea quark masses $m_{u}=m_{d}=0.05 m_{s}$. So far we have 

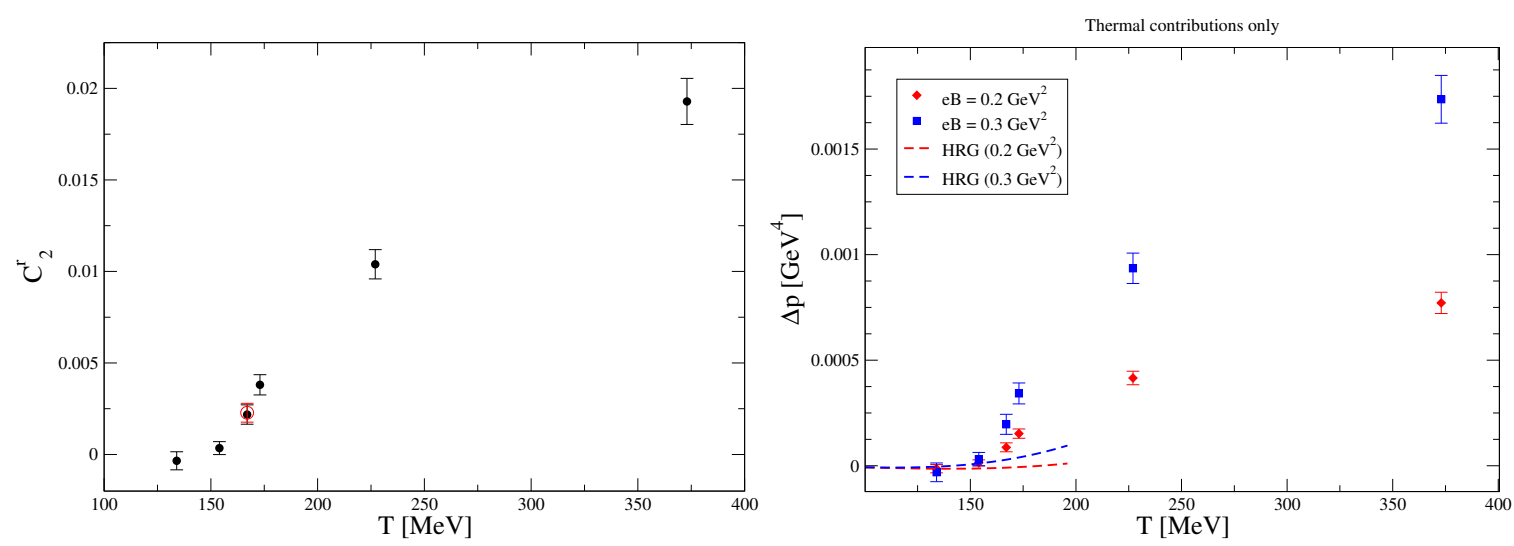

Figure 1: Left panel: second order Taylor expansion coefficient as a function of temperature. The red square is calculated at a larger volume and shows no significant finite volume dependence. Right panel: incremental change in pressure as a function of temperature for various external magnetic fields. The dashed lines show the hadron resonance gas values from [9]. They are computed to all orders in a Taylor expansion.

calculated terms in the presssure expansion only up to $O\left(B^{2}\right)$, i.e. the susceptibility. We have used only 50-70 gauge configurations in each case. Results are tabulated in Table 4. These results were obtained at a cost of about 30K GPU-hours.

The incremental change in pressure is shown as a function of temperature in Fig. 1. Note that $\Delta p$ is a rather small fraction (no more than a few percent) of the zero field pressure for fields up to $O\left(10^{15} \mathrm{~T}\right) \approx 0.1 \mathrm{GeV}^{2} / e$ relevant for heavy-ion collision experiments. For fields as high as $O\left(10^{16} \mathrm{~T}\right) \approx 1 \mathrm{GeV}^{2} / e, 20 \%$ or more.

\section{Acknowledgments}

We are grateful to Urs Heller and Gergely Endrödi for helpful discussions. Computations for this work were carried out with resources provided by the USQCD Collaboration, the University of Utah Center for High Performance Computing, and Indiana University. This work was supported in part by the U.S National Science Foundation under grant PHY10-67881 and the U.S. Department of Energy under grant DE-FC02-12ER-41879.

\section{References}

[1] M. D’Elia, S. Mukherjee and F. Sanfilippo, Phys. Rev. D 82, 051501 (2010) [arXiv: 1005.5365 [hep-lat] ].

[2] M. D'Elia and F. Negro, Phys. Rev. D 83, 114028 (2011) [arXiv:1103.2080 [hep-lat] ].

[3] Plenary talk by Kálmán Szabó.

[4] G. S. Bali, F. Bruckmann, G. Endrodi, Z. Fodor, S. D. Katz, S. Krieg, A. Schafer and K. K. Szabo, JHEP 1202, 044 (2012) [arXiv:1111.4956 [hep-lat] ].

[5] G. S. Bali, F. Bruckmann, G. Endrodi, Z. Fodor, S. D. Katz, S. Krieg, A. Schafer and K. K. Szabo, PoS(LATTiCE 2011)192(2012) [arXiv:1111.5155 [hep-lat]]. 
[6] G. S. Bali, F. Bruckmann, M. Constantinou, M. Costa, G. EndrÃ Či, Z. Fodor, S. D. Katz and S. Krieg et al., PoS (Confinement X) 198 (2012) [arXiv:1301.5826 [hep-lat]].

[7] E. -M. Ilgenfritz, M. Kalinowski, M. Muller-Preussker, B. Petersson and A. Schreiber, Phys. Rev. D 85, 114504 (2012) [arXiv:1203.3360 [hep-lat]].

[8] L. Levkova and C. DeTar, arXiv:1309.1142 [hep-lat].

[9] G. Endrödi, JHEP 1304, 023 (2013) [arXiv:1301.1307 [hep-lat] ].

[10] A. Bazavov, T. Bhattacharya, M. Cheng, C. DeTar, H. T. Ding, S. Gottlieb, R. Gupta and P. Hegde et al., Phys. Rev. D 85, 054503 (2012) [arXiv:1111.1710 [hep-lat] ]. 